\title{
Isolement et caractérisation morphologique de moisissures productrices de substances antibactériennes à partir d'aliments locaux au Burkina Faso
}

\author{
Hamidou COMPAORE ${ }^{1,2 *}$, Hagrétou SAWADOGO-LINGANI ${ }^{2}$, Aly SAVADOGO ${ }^{1}$, \\ Dayéri DIANOU ${ }^{3}$ et Alfred S. TRAORE ${ }^{1}$
}

${ }^{1}$ Université de Ouagadougou, Centre de Recherche en Sciences Biologiques, Alimentaires et Nutritionnelles (CRSBAN), 03 BP 7021 Ouagadougou 03, Burkina Faso.

${ }^{2}$ Département Technologie Alimentaire (DTA/IRSAT/CNRST), 03 BP 7047 Ouagadougou 03, Burkina Faso.

${ }^{3}$ Centre National de Recherches Scientifiques et Technologiques/Institut de Recherche en Sciences de la Santé (IRSS/CNRST), 03 BP 7192 Ouagadougou 03, Burkina Faso.

*Auteur correspondant ; E-mail : hamidoucom@yahoo.fr; (+226) 76360809

\section{RÉSUMÉ}

En vue du risque augmenté jour après jour des bactéries résistantes aux antibiotiques, nous nous sommes intéressés dans cette étude à l'isolement et à la caractérisation des isolats de moisissures productrices d'antibiotique à partir d'aliments locaux du Burkina Faso. Cinquante (50) isolats fongiques isolés ont servis au test d'antibiose qui a révélé vingt (20) isolats producteurs de substances antimicrobiennes. Ce test a concerné les cellules des isolats ainsi que des extraits aqueux et organiques issues de ceux-ci. Trois (03) isolats ont présenté des diamètres d'inhibitions moyens de $20 \mathrm{~mm}$ sur les germes tests. Leur caractérisation a porté sur les critères morphologiques et culturaux. L'isolat $S_{1}$ présente en 5 jours d'incubation sur la gélose de Pomme de Terre Dextrose (PDA) une colonie plate, de couleur blanche au recto, avec un revers jaune, l'isolat $S_{2}$ une colonie vert-olive, de texture veloutée, plus dense au centre et l'isolat $S_{3}$ une petite colonie de couleur verte et ronde. En microscopie optique les isolats $S_{1}$ et $S_{2}$ ont présenté des conidies rondes, réfringentes et nombreuses, les conidiophores sont hyalins, longs et non cloisonnés. L'isolat $S_{3}$ a présenté des conidies rares, des pénicilles constitués de phialides branchés directement à l'extrémité des conidiophores cloisonnés. L'analyse de ces caractéristiques indique que $\mathrm{S}_{1}, \mathrm{~S}_{2}$ et $\mathrm{S}_{3}$ appartiennent respectivement aux genres Aspergillus et Penicillium. (C) 2016 International Formulae Group. All rights reserved.

Mots clés : Substances antimicrobiennes, Aspergillus, Penicillium, Burkina Faso.

\section{Isolation and morphological characterization of fungi producing antibacterial substances from local food in Burkina Faso}

\begin{abstract}
Bacterial resistance to the actual antibiotic is mostly in dangerous progress, why we have interest in isolation and characterization of fungi strains producing antibiotics using local food in Burkina Faso. Fifty (50) isolated strains were used in the antibiosis trial which revealed twenty (20) strains producing antimicrobial substances. This trial concerned the strains cell as well as its aqueous and organic extracts. Three (03) strains had average inhibition diameters of $20 \mathrm{~mm}$ on the testing germs. Their characterization focused on the
\end{abstract}


morphological and culture criteria. After five days of incubation in Potato Dextrose Agar (PDA), the strain $\mathrm{S}_{1}$ shows a flat colony, white on the front, with a yellow reverse. The strain $\mathrm{S}_{2}$ shows an olive-green colony, velvety texture, more dense in the center. As for the strain $\mathrm{S}_{3}$, it shows a round green colored colony. In optical microscopy, strains $S_{1}$ and $S_{2}$ showed numerous round and refractive conidia, conidiophores are hyaline, long and not partitioned. $S_{3}$ strain showed uncommon conidia, penicillus made of phialides which are directly linked to the end of partitioned conidiophores. Analysis of these characteristics indicates that $S_{1}, S_{2}$ and $S_{3}$ belong respectively to the Aspergillus and Penicillium genera.

(C) 2016 International Formulae Group. All rights reserved

Keywords: Antimicrobial substances, Aspergillus, Penicillium, Burkina Faso.

\section{INTRODUCTION}

Depuis l'avènement des antibiotiques, une nette amélioration de la qualité et de la durée de vie a été constatée. Cependant, leur utilisation intensive a eu pour conséquence l'adaptation des bactéries à ces remarquables substances (Boughachiche et al., 2011). En effet, ces dernières années ont été marquées par une augmentation inquiétante de la multi résistance de bactéries pathogènes (Tchamba et al., 2014), la résurgence de maladies infectieuses que l'on croyait parfaitement maîtrisées et l'émergence de nouveaux pathogènes et ceci particulièrement dans les pays en développement en général et ceux d'Afrique Subsaharienne en particulier, en raison de l'inexistence de réglementation et/ou de contrôle (Diande, 2010; Savadogo et Traoré, 2011; Bagré et al., 2015). Ces constats expliquent l'urgence de disposer de nouvelles molécules d'antibiotiques (Boughachiche et al., 2011). L'utilisation abusive des antibiotiques offre des conditions favorables à l'émergence, à la propagation et à la persistance de microorganismes résistants (Savadogo et Traoré, 2011; Bagré et al., 2015). L'OMS dénonce une menace devenue réalité et appelle à une action commune et d'urgence des acteurs concernés contre l'antibiorésistance (Bagré et al., 2015).

La résistance accrue aux antibiotiques classiques des germes pathogènes à l'origine d'infections hospitalières pose un problème de santé publique de premier ordre de nos jours (Boughachiche et al., 2011). A cela s'ajoute le fait que les antibiotiques sont absolument indispensables pour le traitement de la quasitotalité des maladies infectieuses amenant la plupart des industries pharmaceutiques à orienter leurs recherches vers deux axes principaux : la recherche de nouveaux médicaments et le contrôle des phénomènes de résistance des bactéries aux antibiotiques (Olaitan et al., 2014). Ce travail s'inscrit dans le premier axe. Les antibiotiques peuvent être produits par synthèse industrielle ou élaborés par des champignons et diverses bactéries. La technique biologique qui semble être la moins onéreuse et la plus écologique a donc retenu notre attention.

\section{MATERIEL ET METHODES Echantillonnage}

Les prélèvements ont été effectués d'avril 2013 à juin 2013 à divers endroits de la ville de Ouagadougou. Quatre-vingt (80) échantillons d'aliments locaux composés de riz, to, haricot, dèguè, "gonré », sauce pâte d'arachide, mangue, banane, pomme de terre, pain ont été prélevés dans les conditions aseptiques avec des spatules stériles et acheminés au laboratoire dans une glacière à moins de $15{ }^{\circ} \mathrm{C}$. Une aliquote de cinq grammes de chaque aliment a été conservée au congélateur à $-20^{\circ} \mathrm{C}$ pour les analyses.

\section{Isolement et purification des isolats de moisissures}

L'isolement des moisissures à partir des échantillons d'aliments locaux a été fait en utilisant la méthode d'ulster ou la méthode directe (Botton et al., 1990). Elle consiste à 
déposer directement sur le milieu gélosé, les aliquotes d'aliments préalablement conservés au congélateur à $-20{ }^{\circ} \mathrm{C}$. Les milieux de cultures utilisés étaient la gélose Sabouraud et la gélose de Pomme de terre Dextrose (PDA). Après stérilisation à $121{ }^{\circ} \mathrm{C}$ pendant 15 minutes, ces milieux ont été refroidis à $45^{\circ} \mathrm{C}$ et coulés dans des boîtes de Pétri stériles. (Kra et al., 2011; Djabali et Barkat, 2013).

La purification des isolats fongiques a été effectuée par repiquages successifs en points par épuisement des moisissures poussées sur la gélose de Pomme de terre Dextrose (PDA) et la gélose Sabouraud selon la méthode décrite par Ouattara-Sourabie et al. (2011). Les dernières colonies poussées sur les points de repiquages successifs par épuisement ont constitué les isolats purs. Trois repiquages ont été nécessaires pour obtenir des colonies pures.

\section{Matériel bactérien}

Les souches de bactéries pathogènes de références ayant servi au test d'antibiose ont été gracieusement offertes par le Centre Hospitalier Universitaire Yalgado Ouédraogo (CHU-YO). Ce sont: Enterococcus faecalis CIP 10907, Proteus mirabilis ATCC 35659, Shigella dysenteriae CIP 5451, Staphylococcus aureus ATCC 9144, Listeria monocytogenes CRBIB 13134, Pseudomonas aeruginosa ATCC 19249, Salmonella Typhymirium ATCC 13311, Salmonella Enterica CIP 105150, Escherichia coli CIP 105182, Enterobacter aerogenes CIP 104725.

Détermination de la capacité des isolats de moisissures à produire des substances antimicrobiennes

La production des antibiotiques fait appel aux techniques de fermentation qui ont été conduites à $25{ }^{\circ} \mathrm{C}$ dans des erlenmeyers selon les méthodes décrites par Chabasse et al. (2002) et Boughachiche et al. (2011). Les isolats fongiques ont été repiqués sur la gélose de Pomme de terre Dextrose (PDA) et incubées pendant 72 heures à $25^{\circ} \mathrm{C}$. Ainsi ils seront en phase de croissance exponentielle comme suggéré par Sanou (2002).

Test préliminaire d'inhibition: Dans cette partie du travail, l'objectif a été de se focaliser sur le potentiel producteur des activités antimicrobiennes extracellulaires par les isolats fongiques sur milieu solide et en milieu liquide. Un screening primaire a été réalisé afin d'apprécier les activités antimicrobiennes extracellulaires par la méthode des cylindres d'agar contre les germes tests. Ensuite, les isolats fongiques ayant révélé un potentiel producteur de substances antimicrobiennes ont subi un screening secondaire par la méthode des disques en utilisant le filtrat et les extraits organiques issus de la fermentation liquide des isolats fongiques sur l'ensemble des germes tests comme suggérés par Kholkhal (2005) et Aissaoui (2013).

Le screening primaire: L'activité antimicrobienne des isolats fongiques a été testée par la méthode des cylindres d'agar. Les isolats de moisissures ont été ensemencés sur la gélose de Pomme de terre Dextrose (PDA) par écouvillon afin d'obtenir un tapis et incubés jusqu'à l'apparition des spores à une température de $25{ }^{\circ} \mathrm{C}$ (Aissaoui, 2013). Des disques d'agar d'environ $5 \mathrm{~mm}$ de diamètre ont été découpés et déposés sur le milieu Mueller Hinton préalablement ensemencé par une suspension des bactéries tests dont la turbidité a été ajustée par rapport à une solution de 0,5 Mac Farland comme suggéré par Saadoun et al. (1999). Les boîtes sont incubées à $37^{\circ} \mathrm{C}$ pendant 24 heures. Les diamètres des zones d'inhibition ont été mesurés au millimètre près.

Le screening secondaire: Les isolats de moisissures ont été ensemencés sur la gélose de Pomme de terre Dextrose (PDA) et incubés pendant 72 heures à $25^{\circ} \mathrm{C}$. Ils ont été ensuite repiqués dans $50 \mathrm{ml}$ de milieu de fermentation composé de lactosérum enrichi à l'amidon (10 $\mathrm{g} / \mathrm{l})$, extrait de levure (4 g/l), $\mathrm{NH}_{4} \mathrm{Cl}(4 \mathrm{~g} / \mathrm{l}), 2$ $\mathrm{ml}$ de solution d'oligo-éléments, contenus 
dans des erlenmeyers de $250 \mathrm{ml}$ et incubés dans les mêmes conditions pendant 5 jours.

Extraction et concentration des substances antimicrobiennes: Ces substances sont ensuite extraites puis concentrées selon la méthode décrite par Kholkhal (2005) et le test d'antibiose a été réalisé périodiquement toutes les 24 heures du temps de fermentation. Etant donné que la nature de la substance antimicrobienne est inconnue (osidique, peptidique, lipidique ou associée), on a opéré à l'aide de solvants formant trois phases (une phase polaire, une phase semi-polaire et une phase organique). Selon la nature de l'antibiotique, il pourra se retrouver dans l'une des phases. Les solvants organiques utilisés étaient composés : d'éthanol, d'acétate d'éthyle et d'éther de pétrole. La technique d'extraction utilisée est celle dite liquideliquide comme dans le cas de l'extraction des lipopeptides. L'extraction réalisée est de type analytique. Cette méthode a été inspirée de la méthode décrite par Ravindra et al. (2004). L'antibiotique produit a été extrait du milieu de culture à partir du filtrat obtenu (Smaoui, 2010). Cinq grammes du mycélium ont été broyés dans $10 \mathrm{ml}$ de solvants organiques par un broyeur, la phase liquide a été séparée du culot par centrifugation à $10000 \mathrm{rpm}$ pendant 20 minutes. Cette opération a été répétée trois fois en additionnant $10 \mathrm{ml}$ du solvant au culot récupéré. Le filtrat a été déshydraté, par passage sur sulfate de sodium anhydre $\left(\mathrm{Na}_{2} \mathrm{SO}_{4}\right)$, puis évaporé à une température de $45{ }^{\circ} \mathrm{C}$ avec une vitesse de rotation de 150 tours/mn grâce à un évaporateur rotatif. Le résidu obtenu est conservé à $4{ }^{\circ} \mathrm{C}$ comme suggéré par Aissaoui (2013).

Test d'antibiose: Le résidu de substances antimicrobiennes a d'abord été solubilisé dans $5 \mathrm{ml} \mathrm{du}$ même solvant d'extraction. Le test d'antibiose est réalisé avec le filtrat, les extraits organiques concentrés et non concentrés. Des disques blancs d'antibiotiques de $5 \mathrm{~mm}$ de diamètre sont imbibés par $100 \mu \mathrm{l}$ de l'échantillon d'antibiotique (filtrat et/ou extrait), puis séchés 15 minutes dans des boîtes de Pétrie stériles. Ils sont ensuite disposés à la surface de la gélose de Müeller Hinton préalablement ensemencée par les bactéries tests selon la méthode de Bitsi (2002) et Aissaoui (2013). Les boîtes ont été ensuite laissées pendant 2 heures à $4{ }^{\circ} \mathrm{C}$ (pour permettre la diffusion des activités antimicrobiennes) puis incubées à $37{ }^{\circ} \mathrm{C}$ pendant 24 heures. Les diamètres des zones d'inhibition ont été mesurés par la suite. Des disques témoins comportant les solvants d'extraction purs : l'éthanol, l'éther de pétrole et l'acétate d'éthyle sont également testés après séchage afin d'apprécier leur toxicité vis-à-vis des germes tests comme suggérés par Kholkhal (2005) et Boughachiche et al. (2011).

\section{Caractérisation et identification sommaires des isolats d'intérêt}

La caractérisation et l'identification sommaires des isolats d'intérêt ont été faites à l'échelle du genre, à partir des caractères culturaux, morphologiques et la capacité à produire des substances antimicrobiennes selon les techniques utilisées par Bouchet (2005); Ouattara-Sourabie et al. (2011). Les caractéristiques morphologiques des colonies ont été déterminées avec une culture de 5 jours incubée à $25{ }^{\circ} \mathrm{C}$ dans la gélose de Pomme de terre Dextrose (PDA) et dans la gélose sabouraud. La morphologie des cellules (forme, taille, couleur, aspect, contour) a été complétée par un examen microscopique effectué entre lame et lamelle/scotch des cultures ayant servi à l'étude des caractéristiques culturales (Guiraud, 2003).

\section{RÉSULTATS}

Caractéristiques morphologiques des isolats de moisissures collectées

$\mathrm{Au}$ total, vingt (20) isolats de moisissures productrices de substances antimicrobiennes ont été révélés par la 
méthode directe et collectés. Trois (3) isolats fongiques isolés du riz ont présenté les diamètres d'inhibition les plus élevés sur les germes tests et ont été sélectionnés pour la caractérisation et l'identification à l'échelle genre. Dès l'apparition du mycélium, les caractéristiques morphologiques des trois isolats fongiques $\left(\mathrm{S}_{1}, \mathrm{~S}_{2}, \mathrm{~S}_{3}\right)$ telles que la vitesse de croissance, l'aspect, la forme et la couleur des colonies fongiques ont été appréciées à l'œil nu et au microscope optique. Ces caractéristiques sont résumées dans les Tableaux 1 et 2 . Les isolats $S_{1}$ et $S_{2}$ ont présenté de larges colonies de plus de 30 millimètres de diamètre en cinq jours d'incubation à $25{ }^{\circ} \mathrm{C}$ sur la gélose de Pomme de Terre Dextrose (PDA) tandis que l'isolat $\mathrm{S}_{3}$ a présenté de petites colonies rondes de 15 millimètres de diamètre (Tableau 1). Au microscope optique les isolats $S_{1}$ et $S_{2}$ ont présenté de nombreuses conidies réfringentes avec des conidiophores non cloisonnés. L'isolat $S_{3}$ a présenté de rares conidies portées par des conidiophores cloisonnés (Tableau 2).

\section{Identification des isolats de moisissures à l'échelle genre}

L'identification des genres fongiques a été réalisée essentiellement selon les clefs de détermination de Botton et al. (1990), Guiraud (2003) ainsi que celles de Chabasse et al. (2002). Au terme de l'analyse mycologique (Tableaux 1 et 2), deux genres différents ont été isolés et identifiés à partir des échantillons du riz analysés à savoir le genre Penicillium et le genre Aspergillus.

\section{Activité antibactérienne}

L'activité antibactérienne des isolats de moisissures a été testée sur des souches de bactéries pathogènes de référence. Le test a concerné la culture des isolats étudiés, le filtrat, les extraits éthanolique, éthérique et d'acétate d'éthyle.

\section{Activité antibactérienne des cellules des isolats de moisissures}

Les tests relatifs à l'activité antibactérienne des cultures de moisissures en milieu solide par la méthode des cylindres d'agar ont révélé l'existence des zones d'inhibitions des cultures bactériennes autour des colonies de moisissures après 24 heures d'incubation. Les résultats obtenus sont consignés dans le Tableau 3. Il ressort des tests d'antibiose que l'activité des moisissures est très variable sur les souches de bactéries pathogènes testées. Seule la substance sécrétée par l'isolat $S_{3}$ est active sur Escherichia coli. Les diamètres des zones d'inhibitions obtenues avec Enterococcus faecalis, Proteus mirabilis, Shigella dysenteriae, Listeria monocytogenes et Pseudomonas aeruginosa sont compris entre 12 et 23 millimètres. Tandis que Staphylococcus aureus, Salmonella Typhymirium, Salmonella Enterica, Escherichia coli et Enterobacter aerogenes ne présentent pas de zone d'inhibition (Tableau 3).

\section{Activité antibactérienne des filtrats}

Les résultats des tests antibactériens réalisés avec les filtrats sont présentés dans le Tableau 4 et la Figure 1. Il ressort que les zones d'inhibitions apparaîssent après 24 heures d'incubation. Chez certaines souches (Proteus mirabilis et Listeria monocytogenes), les diamètres de ces zones atteignent leur valeur maximale après 48 heures d'incubation. Par contre, chez d'autres (Enterococcus faecalis et Escherichia coli), ils augmentent progressivement pour atteindre leur maximum après 72 heures voir 120 heures d'incubation. En outre, l'extrait éthanolique des trois isolats fongiques a montré une activité antibactérienne sur au moins la moitié des germes tests utilisés. L'éthanol constitue donc le meilleur solvant d'extraction de substances antibactériennes produites par les trois isolats (Tableau 4). 


\section{Activité antibactérienne des extraits organiques}

Les extraits organiques (éthanol, éther de pétrole et acétate d'éthyle) obtenus à partir de 24 heures de fermentation en milieu liquide des isolats de moisissures n'ont eu aucun effet d'inhibition sur les germes tests utilisés. Il a donc été nécessaire de prolonger la durée de fermentation à 120 heures. Les extraits obtenus à partir de 72 heures de fermentation ont donné des diamètres d'inhibition sur la moitié des germes tests utilisés. Les résultats sont consignés dans le Tableau 5 et la Figure
2. Les tests antibactériens réalisés avec les extraits éthanolique et éthérique de l'isolat $S_{1}$ montrent une activité de tous ces extraits sur Proteus mirabilis, Pseudomonas aeruginosa, et Shigella dysenteriae (Tableau 5). L'extrait d'acétate d'éthyle n'est actif que sur Listeria monocytogenes. L'extrait éthanolique est actif sur les souches de Salmonella Enterica et Enterococcus faecalis qui sont résistantes aux extraits éthérique et d'acétate d'éthyle. La souche Listeria monocytogenes, résistante à l'extrait éthérique est sensible aux extraits éthanolique et d'acétate d'éthyle (Tableau 5).

Tableau 1: Aspect macroscopique des trois isolats de moisissures sur la gélose de Pomme de terre Dextrose (PDA) incubé à $25^{\circ} \mathrm{C}$ pendant 5 jours.

\begin{tabular}{|c|c|c|}
\hline Isolats & Caractères culturaux des colonies & Colonies \\
\hline $\mathrm{S}_{1}$ & $\begin{array}{l}\text { Colonies blanches crème au recto, arrondies, uniformes avec } \\
\text { sclérose, au centre jaunâtre en forme de cratère, solidement } \\
\text { adhérée à la gélose. Diamètre compris entre } 30 \text { et } 35 \mathrm{~mm} \text {, } \\
\text { envers jaunâtre. }\end{array}$ & \\
\hline $\mathrm{S}_{2}$ & $\begin{array}{l}\text { Colonies vert olive au recto sans sclérose, surélevées, texture } \\
\text { veloutée rayonnantes en bordure, floconneuses, cotonneuses, } \\
\text { plus denses et vert foncé au centre. Diamètre compris entre } 40 \\
\text { et } 55 \mathrm{~mm} \text {, envers jaunâtre. }\end{array}$ & \\
\hline $\mathrm{S}_{3}$ & $\begin{array}{c}\text { Colonies petites et rondes, de couleur vert blanc, solidement } \\
\text { adhérées à la gélose. Diamètre compris entre } 10 \text { et } 15 \mathrm{~mm} \text {, } \\
\text { envers incolore. }\end{array}$ & \\
\hline
\end{tabular}


Tableau 2: Aspect microscopique des trois isolats de moisissures.

\begin{tabular}{|c|c|c|c|}
\hline Isolats & Aspects microscopiques & Genre & Cellules \\
\hline $\mathrm{S}_{1}$ & $\begin{array}{l}\text {-Mycélium hyalin, non cloisonné } \\
\text {-conidiophores nombreux, longs, dréssés et non ramifiés, } \\
\text { terminés en vésicule jaunâtre } \\
\text {-Phialides formés directement sur la vésicule } \\
\text {-Conidies en chaîne divergente }\end{array}$ & $\begin{array}{l}\text { Aspergillus sp } \\
(\mathrm{Gr} \times 100)\end{array}$ & \\
\hline $\mathrm{S}_{2}$ & $\begin{array}{l}\text {-Mycélium hyalin, non cloisonné } \\
\text {-conidiophores courts, lisses, incolores, dréssés et non } \\
\text { ramifiés, terminés en vésicule rosâtre } \\
\text {-Têtes aspergillaires radiaires } \\
\text {-Conidies en chaîne divergente }\end{array}$ & $\begin{array}{l}\text { Aspergillus sp } \\
(\mathrm{G} \times 100)\end{array}$ & \\
\hline $\mathrm{S}_{3}$ & $\begin{array}{l}\text {-Conidiophores isolés et dréssés } \\
\text {-Pénicilles constitués de phialides branchés directement à } \\
\text { l'extrémité des conidiophores cloisonnés }\end{array}$ & $\begin{array}{l}\text { Penicillium sp } \\
(\mathrm{G} \times 100)\end{array}$ & \\
\hline
\end{tabular}

Tableau 3: Résultat de l'activité antibactérienne des isolats de moisissures contre les souches de bactéries pathogènes de référence par la technique des cylindres d'agar.

\begin{tabular}{lccc}
\hline \multirow{2}{*}{ Bactéries pathogènes de référence } & \multicolumn{3}{c}{ Diamètre d'inhibition (mm) } \\
\cline { 2 - 4 } & Isolat 1 & Isolat 2 & Isolat 3 \\
\hline Enterococcus faecalis CIP 10907 & 12 & 6 & 0 \\
Proteus mirabilis ATCC 35659 & 16 & 12 & 13 \\
Shigella dysenteriae CIP 5451 & 12 & 16 & 9 \\
Staphylococcus aureus ATCC 9144 & 0 & 0 & 0 \\
Listeria monocytogenes CRBIB 13134 & 23 & 20 & 26 \\
Pseudomonas aeruginosa ATCC 19249 & 16 & 18 & 17 \\
Salmonella Typhymirium ATCC 13311 & 0 & 0 & 0 \\
Salmonella Enterica CIP 105150 & 0 & 0 & 0 \\
Escherichia coli CIP 105182 & 0 & 0 & 6 \\
Enterobacter aerogenes CIP 104725 & 0 & 0 & 0 \\
\hline
\end{tabular}

Diamètres d'inhibition $(\mathrm{mm})$ mesurées après 24 heures et 48 heures d'incubation à $37{ }^{\circ} \mathrm{C}$ sur le milieu Mueller Hinton $\mathrm{pH} 7,4$. 
Tableau 4: Résultats de l'activité antibactérienne du filtrat contre les souches de bactéries pathogènes de référence par la technique des disques.

\begin{tabular}{lccccccccc}
\hline & \multicolumn{3}{c}{ Isolat 1 } & \multicolumn{3}{c}{ Isolat 2 } & \multicolumn{3}{c}{ Isolat 3 } \\
\hline Bactéries & $\mathbf{2 4}$ & $\mathbf{4 8}$ & $\mathbf{7 2}$ & $\mathbf{2 4}$ & $\mathbf{4 8}$ & $\mathbf{7 2}$ & $\mathbf{2 4}$ & $\mathbf{4 8}$ & 72 \\
\hline Enterococcus faecalis CIP 10907 & 10 & 10 & 14 & 5 & 6 & 10 & 0 & 0 & 0 \\
Proteus mirabilis ATCC 35659 & 12 & 12 & 12 & 9 & 14 & 14 & 10 & 10 & 12 \\
Shigella dysenteriae CIP 5451 & 9 & 14 & 15 & 12 & 12 & 12 & 10 & 10 & 10 \\
Staphylococcus aureus ATCC 9144 & 0 & 0 & 0 & 0 & 0 & 0 & 0 & 0 & 0 \\
Listeria monocytogenes CRBIB 13134 & 19 & 19 & 19 & 16 & 17 & 21 & 25 & 25 & 25 \\
Pseudomonas aeruginosa ATCC 19249 & 18 & 18 & 18 & 20 & 21 & 22 & 18 & 18 & 18 \\
Salmonella Typhymirium ATCC 13311 & 0 & 0 & 0 & 0 & 0 & 0 & 0 & 0 & 0 \\
Salmonella Enterica CIP 105150 & 0 & 0 & 0 & 0 & 0 & 0 & 0 & 0 & 0 \\
Escherichia coli CIP 105182 & 0 & 0 & 0 & 0 & 0 & 0 & 10 & 10 & 12 \\
Enterobacter aerogenes CIP 104725 & 0 & 0 & 0 & 0 & 0 & 0 & 0 & 0 & 0 \\
\hline
\end{tabular}

Diamètre d'inhibition (en mm) du filtrat de culture de moisissure mesuré après 24,48 et 72 heures d'incubation à $37^{\circ} \mathrm{C}$ sur le milieu Mueller Hinton pH 7,4.

Tableau 5: Résultats de l'activité antibactérienne des extraits éthanolique (A), éthérique (B) et de l'acétate d'éthyle (C) du filtrat obtenu par fermentation des moisissures contre les souches de bactéries pathogènes de référence.

\begin{tabular}{|c|c|c|c|c|c|c|c|c|c|c|}
\hline \multirow{3}{*}{ Bactérie } & \multicolumn{10}{|c|}{ Diamètre d'inhibition (mm) } \\
\hline & \multirow{2}{*}{$\begin{array}{l}\text { Moisissure } \\
\text { Extrait }\end{array}$} & \multicolumn{3}{|c|}{ Isolat 1} & \multicolumn{3}{|c|}{ Isolat 2} & \multicolumn{3}{|c|}{ Isolat 3} \\
\hline & & $\mathbf{A}$ & $\mathbf{B}$ & $\mathbf{C}$ & $\mathbf{A}$ & B & $\mathbf{C}$ & $\mathbf{A}$ & B & $\mathrm{C}$ \\
\hline \multicolumn{2}{|c|}{ Enterococcus faecalis CIP 10907} & 12 & 0 & 0 & 7 & 0 & 0 & 0 & 0 & 0 \\
\hline \multicolumn{2}{|c|}{ Proteus mirabilis ATCC 35659} & 16 & 13 & 0 & 9 & 0 & 0 & 10 & 13 & 0 \\
\hline \multicolumn{2}{|c|}{ Shigella dysenteriae CIP 5451} & 9 & 14 & 0 & 12 & 0 & 0 & 0 & 12 & 0 \\
\hline \multicolumn{2}{|c|}{ Staphylococcus aureus ATCC 9144} & 0 & 0 & 0 & 0 & 0 & 0 & 0 & 0 & 0 \\
\hline \multicolumn{2}{|c|}{ Listeria monocytogenes CRBIB 13134} & 20 & 0 & 7 & 20 & 0 & 0 & 25 & 23 & 0 \\
\hline \multicolumn{2}{|c|}{ Pseudomonas aeruginosa ATCC 19249} & 22 & 10 & 0 & 24 & 0 & 0 & 20 & 12 & 6 \\
\hline \multicolumn{2}{|c|}{ Salmonella Typhymirium ATCC 13311} & 0 & 0 & 0 & 0 & 0 & 0 & 0 & 0 & 0 \\
\hline \multicolumn{2}{|c|}{ Salmonella Enterica CIP 105150} & 12 & 0 & 0 & 0 & 0 & 0 & 7 & 10 & 0 \\
\hline \multicolumn{2}{|c|}{ Escherichia coli CIP 105182} & 0 & 0 & 0 & 0 & 0 & 0 & 14 & 0 & 0 \\
\hline \multicolumn{2}{|c|}{ Enterobacter aerogenes CIP 104725} & 0 & 0 & 0 & 0 & 0 & 0 & 0 & 0 & 0 \\
\hline
\end{tabular}

Diamètre d'inhibition des extraits mesurée après 72 heures de fermentation à $30^{\circ} \mathrm{C}$. A : Extrait éthanolique ; B : Extrait éthérique ; $\mathrm{C}$ : Extrait d'acétate d'éthyle.
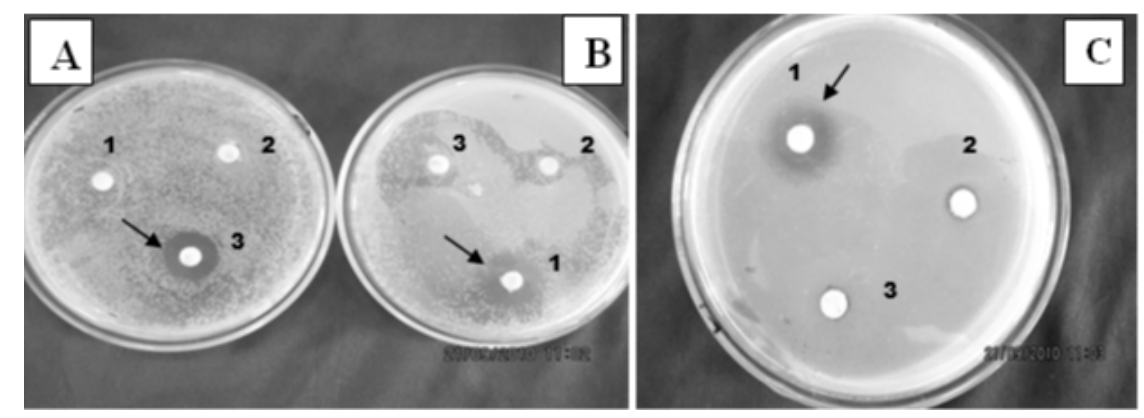

Figure 1: Résultat du test d'antibiose du filtrat. A : Isolat $S_{3}$ sur Escherichia coli, B : Isolat $S_{1}$ sur Shigella dysenteriae, $\mathrm{C}$ : Isolats $\mathrm{S}_{1}$ et $\mathrm{S}_{2}$ sur Enterococcus faecalis. 

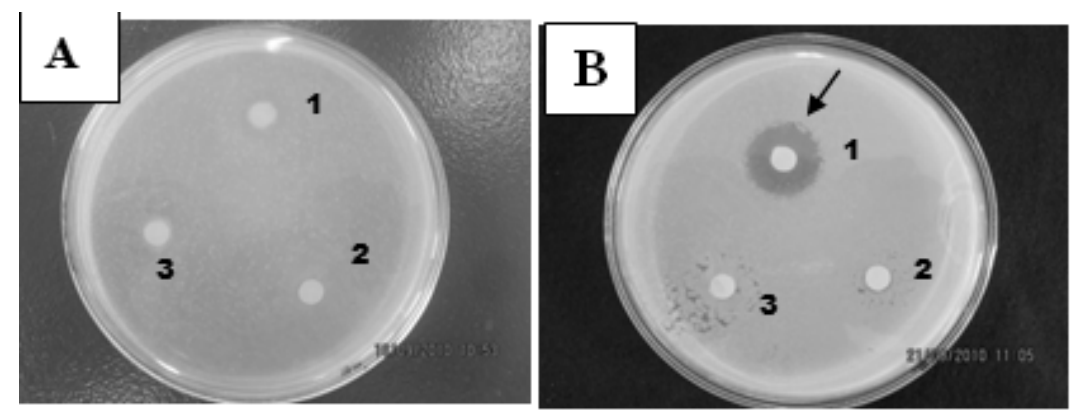

Figure 2: Souche de Staphylococcus aureus devenue sensible à la substance sécrétée par l'isolat $\mathrm{S}_{3}$ après extraction et concentration. (A) : culture de Staphylococcus aureus en présence des extraits organiques non concentré de l'isolat $\mathrm{S}_{3}:$ (B) : culture de Staphylococcus aureus en présence de l'extrait éthanolique concentré de l'isolat $\mathrm{S}_{3}$.

\section{DISCUSSION}

Identification des isolats de moisissures à l'échelle genre

Les caractéristiques macroscopiques et microscopiques des isolats $\mathrm{S}_{1}, \mathrm{~S}_{2}$ et $\mathrm{S}_{3}$ sont respectivement semblables à celles des genres Aspergillus et Penicillium (Chabasse et al., 2002). Selon certains auteurs, des moisissures appartenant à ces deux genres sont généralement productrices de substances antibactériennes (Chabasse et al., 2002; Kholkhal, 2005). Au regard de cette information et des similitudes entre les isolats étudiés et les genres décrits par Chabasse et al. (2002) et Ouattara-Sourabie et al. (2011). Les isolats $\mathrm{S}_{1}, \mathrm{~S}_{2}$ et $\mathrm{S}_{3}$ pourraient appartenir respectivement aux genres Aspergillus et Penicillium.

\section{Analyse de l'activité antibactérienne des moisissures}

L'inhibition de la croissance bactérienne par les cultures de moisissures prouve l'élaboration et l'excrétion par les isolats fongiques de substances antimicrobiennes qui diffusent dans le milieu solide (Bitsi, 2002). La durée d'élaboration et d'excrétion de ces substances est plus courte en milieu solide qu'en milieu liquide (Boughachiche et al., 2011). Ceci pourrait s'expliquer par la différence existant au niveau de la composition chimique des milieux et/ou de certains paramètres tels que : la température, le $\mathrm{pH}$ et la nature du substrat.

Les tests d'antibiose réalisés avec les extraits montrent que l'activité des moisissures est très variable sur les souches de bactéries pathogènes testées. Plus de la moitié des germes tests est sensible aux substances antibactériennes produites par les isolats fongiques.

Quelques hypothèses peuvent justifier cette explication :

(i) Les isolats produiraient plusieurs types de substances antimicrobiennes.

(ii) Ces substances montrent une grande solubilité dans les solvants utilisés.

(iii) L'extraction des molécules actives a été plus ou moins totale.

En effet, l'extraction par épuisement a été réalisée. Cinq grammes de mycélium ont été broyés dans $10 \mathrm{ml}$ de solvant, le mélange a été centrifugé, puis la phase organique est pipetée, et $10 \mathrm{ml}$ du même solvant ont été rajoutés dans le culot. La même opération a été répétée trois fois, puis les phases organiques ont été collectionnées et ensuite concentrées.

Les tests d'antibiose réalisés avec les différents extraits montrent que quatre des germes tests sont en même temps sensibles 
aux extraits éthanolique et éthérique des isolats $S_{1}$ et $S_{2}$. Les six autres germes tests sont seulement sensibles à au plus un extrait des isolats fongiques $S_{1}, S_{2}$ et $S_{3}$. Ces résultats pourraient s'expliquer par l'hypothèse selon laquelle les principes actifs agissant sur Proteus mirabilis, Pseudomonas aeruginosa et Shigella dysenteriae sont en même temps présents dans les extraits éthanolique et éthérique en concentration suffisante (El Amri et al., 2014; Kouadio et al., 2015). Le principe actif agissant sur Salmonella Enterica et Enterococcus faecalis est absent dans les extraits éthérique et d'acétate d'éthyle ou s'y trouverait à des concentrations trop faibles pour provoquer une inhibition (Bitsi, 2002).

On peut émettre l'hypothèse d'une existence de principes actifs (ou d'antibiotique) de nature différente dans le filtrat. Certains de ces principes peuvent être extraits à la fois par les phases éthanolique et éthérique ou par l'une d'elles. D'autres par contre sont extractibles uniquement par l'acétate d'éthyle (Devi et al., 2007; Kouadio et al., 2015).

Les tests antibactériens réalisés avec les extraits éthanolique de l'isolat $S_{3}$ présentent, après concentration, un diamètre d'inhibition de 15 millimètres sur Staphylococcus aureus qui était résistant au filtrat et à l'extrait non concentré (Figure 2). On peut penser que la substance existait dans le filtrat et dans l'extrait non concentré, mais en concentration faible pour provoquer une zone d'inhibition.

Les tests d'antibiose réalisés avec la culture, le filtrat et les différents extraits organiques, suggèrent que les substances antimicrobiennes produits présenteraient un large spectre au regard des isolats bactériennes testées: Cocci Gram+, bacille Gram+ et Gram-. Ces résultats sont concordants avec ceux de Kholkhal (2005) et Devi et al. (2007).

Le procédé d'extraction des antibiotiques utilisé est celui des lipopeptides.
Les tests positifs traduisent que les substances antimicrobiennes seraient composées des lipopeptides et qu'elles sont solubles dans ces solvants (Compaoré, 2011). Les lipopeptides sont des molécules amphiphiles constituées d'une partie peptidique polaire et d'une chaîne hydrocarbonée d'acide gras apolaire. Ils sont dotés de diverses activités antibactériennes (Guiraud, 2003; Romero et al., 2007).

Les tests antibactériens réalisés avec le filtrat et les extraits organiques obtenus après 24 heures de fermentation se sont avérés négatifs. On peut expliquer ces résultats par l'effet glucose. En effet, à cause de son assimilation rapide, le glucose exerce une répression catabolique (effet glucose) chez la plupart des microorganismes producteurs d'antibiotiques qui l'utilisent. Une source de carbone rapidement assimilable exerce une action négative sur la biosynthèse des métabolites secondaires (Bitsi, 2002; Compaoré, 2011). On peut émettre l'hypothèse selon laquelle la fermentation de 24 heures ne permet pas la synthèse de l'antibiotique à cause de la répression catabolique exercée par le glucose non métabolisé encore présent dans le milieu de culture. Cet effet avait été mis en évidence au cours de la production de la pénicilline (Guiraud, 2003). La présence du glucose inhibe la synthèse de la pénicilline bien que favorisant la croissance du microorganisme et du mycélium (Bitsi, 2002). Lorsque l'on utilise un milieu de culture contenant comme source de carbone le glucose et le lactose, la synthèse de la pénicilline ne commence que lorsque le lactose devient la seule source de carbone dans le milieu après assimilation complète du glucose (Bitsi, 2002; Compaoré, 2011). C'est pourquoi certains chercheurs ont proposé pour la biosynthèse de la pénicilline l'utilisation du lactose ou de l'amidon comme source de carbone afin d'éliminer l'effet négatif de la source carbonée sur la biosynthèse. 
La durée de fermentation nécessaire à la production d'un antibiotique est fonction de la nature du microorganisme producteur et de la source de carbone utilisée. Avec le glucose comme source de carbone, cette durée est de deux jours pour la production du chlorotétracycline, de cinq à six jours pour la streptomycine et de trois jours pour la pénicilline (Compaoré, 2011).

Le filtrat obtenu après 72 heures de fermentation et les extraits réalisés à partir de ce dernier ont présenté une activité inhibitrice sur la plupart des germes testés. Ces résultats prouvent l'existence d'une substance antimicrobienne dont la synthèse se réalise dans l'intervalle de temps compris entre 24 heures et 72 heures de fermentation. On peut affirmer que les conditions nécessaires à la synthèse des substances antimicrobiennes par les isolats de moisissures sélectionnées sont réunies dans cet intervalle de temps. Il pourrait s'agir de l'assimilation complète du glucose dont la présence inhibait la synthèse de l'antibiotique.

\section{Conclusion}

Ces travaux ont permis d'isoler et d'identifier à l'échelle du genre trois isolats fongiques dont les propriétés antimicrobiennes ont été mises en évidence. D'une part les caractéristiques morphologiques et culturales des trois (3) isolats fongiques sont connues et d'autre part, les tests antibactériens de la culture sur milieu solide, du filtrat des cultures liquides et des extraits de ces filtrats ont prouvé la synthèse d'une ou de plusieurs molécules antimicrobiennes par les isolats fongiques. L'analyse des caractéristiques morphologiques et culturales ont permis d'affilier aux isolats $S_{1}$ et $S_{2}$ le genre Aspergillus et à l'isolat $\mathrm{S}_{3}$ le genre Penicillium. L'objectif de ce travail qui consistait à isoler à partir d'aliments locaux des isolats de moisissures productrices de substances antibactériennes a été en grande partie atteint. Cependant, pour valoriser ces travaux, il est nécessaire de les poursuivre par l'identification complète des trois isolats ainsi que l'extraction, la purification et la caractérisation des molécules bioactives.

\section{REMERCIEMENTS}

Cette recherche a été initiée dans le cadre de la soutenance d'une thèse de Doctorat unique. Nous remercions le Laboratoire de Microbiologie et de Biotechnologie du CRSBAN ainsi que le laboratoire accrédité de Microbiologie du DTA/IRSAT/CNRST qui nous ont aidés pour le prélèvement, l'analyse des échantillons et l'interprétation des résultats.

\section{CONFLITS D'INTÉRÊTS}

Les auteurs déclarent qu'ils n'ont aucun conflit d'intérêts.

\section{CONTRIBUTION DES AUTEURS}

AST, DD et AS ont contribué à concevoir et à planifier les expériences ; HC les a réalisées. DD, HS-L et AST ont fourni les réactifs, le matériel et les outils d'analyse. HC et HS-L ont écrit le manuscrit. Tous les auteurs ont lu et approuvé la version finale du manuscrit.

\section{REFERENCES}

Aissaoui N. 2013. Etude de molécules d'antibiotiques biosynthétisées par une bactérie extrêmophile issue d'une Sebkha Algérienne d'El Goléa. Thèse de Doctorat, Université de Tlemcen, Tlemcen, p. 174.

Bagré TS, Samandoulougou S, Traoré M, Illy D, Tchamba GB, Bawa HI, Bouda SC, Traoré AS, Barro N. 2015. Détection biologique des résidus d'antibiotiques dans le lait et produits laitiers de vache consommés à Ouagadougou, Burkina Faso. Journal of Applied Biosciences, 87: 
8105- 8112. DOI : http://dx.doi.org/ 10.4314/jab.v87i1.11

Bitsi MJM. 2002. Isolement et caractérisation d'une souche de champignon productrice d'antibiotiques. Diplôme d'études Approndies (DEA), Université de Ouagadougou, Ouagadougou, p. 58.

Botton B, Breton A, Fèvre M, Gauthier. S, Guy P, Larpent J-P, Reymond P, Sanglier J-J, Vayssier Y, Veau P. 1990. Moisissures Utiles et Nuisibles d'Importance Industrielle. Ed. Masson Collection Biotechnologie: Paris; 34-381.

Bouchet P. 2005. Les champignons. Mycologie fondamentale et appliquée (ed). Masson: Paris; 1-25.

Boughachiche F, Reghioua S, Zerizer H, Boulahrouf A. 2011. Activité antibactérienne d'espèces rares de Streptomyces contre des isolats cliniques multirésistants. Ann. Biol. Clin., 70(2): 167-174. DOI : 10.1684/abc.2012.0661

Chabasse D, Bouchra J-P, De Gentile L, Brun S, Cimon B, Penn P. 2002. Les Moisissures d'Intérêt Médical. Cahier de formation $\mathrm{N}^{\circ} 25$, Bioforma : Paris ; 160.

Compaoré H. 2011. Caractérisation des moisissures productrices d'antibiotique et propriétés biochimiques des antibiotiques produits. Diplôme d'Etude Approfondies (DEA), Université de Ouagadougou, Ouagadougou, p. 102.

Devi P, D'Souza L, Kamat T, Rodrigues C, Naik GC. 2007. Batch culture fermentation of Penicillium Chrysogenum and a report on the isolation, purification, identification and antibiotic activity of citrinin. Indian Journal of Marine Sciences, 38(1): 38-44.

Diande S. 2010. Evaluation de la résistance de Mycobacterium tuberculosis aux antibiotiques et exploration des facteurs de risque associés à la multirésistance au Burkina Faso. Thèse de Doctorat,
Université de Ouagadougou, Ouagadougou, p. 117.

Djabali S, Barkat M. 2013. Isolement et identification de moisissures contaminant deux variétés d'haricot sec stockées à température ambiante Rev. Microbiol. Ind. San et Environn., 7(1): 120-132.

El Amri J, Elbadaoui K, Zair T, Bouharb H, Chakir S, Alaoui TI. 2014. Study the antibacterial activity of essential oils Teucrium capitatium $L$ and Silene vulgaris extract on different strains tested. Journal of Applied Biosciences, 82 (1): 7481-7492. DOI http://dx.doi.org/10.4314/jab.v82i1.16

Guiraud J-P. 2003. Microbiologie Alimentaire Ed. Duond: Paris.

Kholkhal W. 2005. Recherche de nouvelles souches fongiques productrices d'antibiotiques à partir du sol et des concrétions sédimentaires des grottes de Aïn Fezza. Mémoire de Magister, Université Abou Bekr Belkaid, Tlemcen, p. 85 .

Kouadio NJ, Guessennd NK, Kone MW, Moussa B, Koffi YM, Guede KB, Yao K, Bakayoko A, Trabi HF, Dosso M. 2015. Evaluation de l'activité des feuilles de Mallotus oppositifolius (Geisel.) Müll.Arg (Euphorbiaceae) sur des bactéries multirésistantes et criblage phytochimique. Int. J. Biol. Chem. Sci., 9(3): 1252-1262. DOI : http://dx.doi.org/10.4314/ijbcs.v9i3.10

Kra KD, Diallo HA, Kobenan K, Kone De, Kouadio YJ. 2011. Diagnostic de la fusariose du bananier sur les cultivars grande naine (Musa AAA) et corne 1 (Musa $\mathrm{AAB}$ ) à la périphérie du District d'Abidjan (Côte d'Ivoire). Int. J. Biol. Chem. Sci., 5(4): 1501-1514. DOI : http://ajol.info/index.php/ijbcs

Olaitan AO, Morand S, Rolain JM. 2014. Mechanisms of polymyxin resistance: acquired and intrinsic resistance in 
bacteria. Frontiers in Microbiology, 26(5): 643-650. DOI : http://dx.doi.org/10.3389/fmicb.2014.006 43

Ouattara-Sourabie PB, Nikiema PA, Traore AS. 2011. Caractérisation de souches d'Aspergillus spp isolées des graines d'arachides cultivées au Burkina Faso, Afrique de l'Ouest. Int. J. Biol. Chem. Sci., 5(3): 1232-1249. DOI : http://ajol.info/index.php/ijbcs

Ravindra G, Ranganayaki RS, Raghothama S, Srinivanan MC, Gilardi RD, Karle IL, Balaram P. 2004. Two novel hexadepsipeptides with several modified amino acid residues isolated from the Fungus Isaria. Chemistry and Biodiversity, 1: 6234-6245. DOI : http://dx.doi.org/10.1039/c1ob05392b

Romero D, de Vicente A, Rakotoaly RH, Dufour SE, Veening J-W, Eva A, Cazorla FM, Kuipers OP, Paquot M, Pérez-García A. 2007. The Iturin and Fengycin families of lipopeptides are key factors in antagonism of Bacillus subtilis toward Podosphaera fusca. The American Phytopathological Society, 20(4): 430440. DOI: 10.1094/MPMI -20-4-0430

Saadoun I, hameed KM, Moussaoui A. 1999. Characterization and analysis of antibiotic activity of some aquatic actinomycetes.
Microbios., 99: 173-179. DOI : http://dx.doi.org/10.1016/S22211691(12)60375

Sanou D. 2002. contribution à la recherche d'antibiotiques à partir des champignons au Burkina Faso « recherche de microorganismes producteurs d'antibiotique ». Diplôme d'Etudes Approfondies (DEA), Université de Ouagadougou, Ouagadougou, p. 91.

Savadogo A, Traoré AS. 2011. La flore microbienne et les propriétés fonctionnelles des yaourts et laits fermentés. Int. J. Biol. Chem. Sci., 5(5): 1-19. DOI : http://ajol.info/index. php/ijbcs

Smaoui S. 2010. Purification et caractérisation de biomolécules à partir de microorganismes nouvellement isolés et identifiés. Thèse de Doctorat, Université de Toulouse, Toulouse, p. 238.

Tchamba GB, Bawa HI, Nzouankeu A, Bagré TS, Dembélé R, Bonkoungou IJO, Zongo C, Savadogo A, Traoré AS, Barro N. 2014. Occurrence and antimicrobial susceptibility of Escherichia coli and Salmonella spp. isolated from "zoomkoom" beverage and ice in Ouagadougou, Burkina Faso. African Journal of Microbiology Research, 8: 3243-3249. DOI : 10.5897/AJMR2014.7014 